\title{
Newly-Diagnosed Disturbed Glucose Metabolism is Associated with Atherosclerosis in Patients with Transient Ischemic Attack or Ischemic Stroke
}

\section{Susanne Fonville ${ }^{1}$, Anouk C van Dijk ${ }^{1,2}$, Taihra Zadi ${ }^{2}$, Evita G van den Herik ${ }^{1}$, Hester F Lingsma ${ }^{3}$, Peter J Koudstaal', Aad van der Lugt ${ }^{2}$ and Heleen M. den Hertog ${ }^{4}$}

${ }^{1}$ Departments of Neurology, Erasmus MC University Medical Center, Rotterdam, Netherlands ${ }^{2}$ Departments of Radiology, Erasmus MC University Medical Center, Rotterdam, Netherlands

${ }^{3}$ Departments of Public Health, Erasmus MC University Medical Center, Rotterdam, Netherlands

${ }^{4}$ Department of Neurology, Medisch Spectrum Twente, Enschede, Netherlands

\begin{abstract}
Objective: Newly-diagnosed disturbed glucose metabolism is highly prevalent in non-diabetic patients with transient ischemic attack (TIA) or ischemic stroke, and increases the risk of recurrent stroke. Diabetes mellitus is associated with atherosclerosis. We aimed to assess whether newly-diagnosed disturbed glucose metabolism is associated with atherosclerosis as well.

Research design and methods: Patients with a recent TIA or ischemic stroke were classified in three groups based on glucose levels and use of antidiabetic drugs. Pre-existent diabetes mellitus was defined as the use of antidiabetic drugs prior to the event. Newly-diagnosed disturbed glucose metabolism was defined as two or more disturbed glucose tests: fasting plasma glucose level $\geq 5.6 \mathrm{mmol} / \mathrm{L}, 2$-hour post-load glucose level $\geq 7.8 \mathrm{mmol} / \mathrm{L}$, and/or glycosylated hemoglobin level $\geq 39 \mathrm{mmol} / \mathrm{mol}$.

We used CT-angiography to assess stenosis in the carotid artery bifurcations and calcification volume in the aortic arch, carotid bifurcations and intracranial carotid arteries. The relation between glucose groups and measures of atherosclerosis was expressed as odds ratios and beta coefficients with corresponding $95 \% \mathrm{Cl}$, adjusted for potential confounders.
\end{abstract}

Results: Of the 1217 patients, 384 (32\%) had newly-diagnosed disturbed glucose metabolism, and $210(17 \%)$ had pre-existent diabetes mellitus. Newly-diagnosed disturbed glucose metabolism was independently associated with stenosis $\geq 50 \%(\mathrm{aOR}(95 \% \mathrm{Cl}) 1.15(1.04-2.20))$. Pre-existent diabetes mellitus was associated with stenosis $\geq$ $50 \%$ and with calcification volume in all regions, especially in patients without the use of cholesterol-lowering drugs prior to the event.

Conclusions: Our study shows that newly-diagnosed disturbed glucose metabolism in patients with a recent TIA or ischemic stroke is associated with more severe extra- and intracranial atherosclerosis, similar to pre-existent diabetes mellitus.

Keywords: Stroke, atherosclerosis, glucose, pre-diabetes

\section{Introduction}

Diabetes mellitus is a well-known risk factor for first stroke and stroke recurrence [1]. Pre-diabetes is a metabolic state with a high risk of developing diabetes mellitus in the future [2,3] and comprises impaired fasting glucose and/or impaired glucose tolerance and/ or disturbed glycosylated hemoglobin levels [3,4]. Pre-diabetes and newly-diagnosed diabetes mellitus are highly prevalent (up to 79\%) in patients with an ischemic stroke or transient ischemic attack (TIA) without known diabetes mellitus prior to the event [5-9] and is also associated with an increased risk of cardiovascular disease and recurrent stroke [10-12].

Patients with diabetes mellitus are highly susceptible for atherosclerosis, which is reflected in increased carotid intima media thickness (CIMT) [13-15] and severe calcification in the aortic arch and carotid arteries [16,17]. However, it is still unsure whether the association between diabetes mellitus and calcification volume is similar between men and women [16-20]. Diabetes mellitus is characterized by a clustering of vascular risk factors, including dyslipidemia, hypertension, obesity, and insulin resistance. Among these factors, dyslipidemia may play an important role in the development of atherosclerosis [21,22]. More severe atherosclerosis is presumed to be the main underlying mechanism of the increased risk of recurrent stroke in patients with diabetes mellitus. However, there is no consensus whether pre-diabetes is associated with atherosclerosis as well $[13-15,23,24]$.

To the best of our knowledge, there are no studies on the association between newly-diagnosed disturbed glucose metabolism (pre-diabetes or newly-diagnosed diabetes mellitus) and atherosclerosis in patients with TIA or ischemic stroke. We therefore aimed to assess whether

*Corresponding author: Susanne Fonville, Erasmus MC University Medical Center Department of Neurology's, Gravendijkwal 230, 3015 CE Rotterdam, Netherlands, Tel: +31107044206; Fax: +31107042270; E-mail: s.fonville@erasmusmc.nl

Received December 09, 2014; Accepted January 22, 2015; Published January 28, 2015

Citation: Fonville S, van Dijk AC, Zadi T, van den Herik EG, Lingsma HF, et al (2015) Newly-Diagnosed Disturbed Glucose Metabolism is Associated with Atherosclerosis in Patients with Transient Ischemic Attack or Ischemic Stroke. J Diabetes Metab 6: 496. doi:10.4172/2155-6156.1000496

Copyright: $\odot 2015$ Fonville S, et al. This is an open-access article distributed unde the terms of the Creative Commons Attribution License, which permits unrestricted use, distribution, and reproduction in any medium, provided the original author and source are credited. 
Citation: Fonville S, van Dijk AC, Zadi T, van den Herik EG, Lingsma HF, et al. (2015) Newly-Diagnosed Disturbed Glucose Metabolism is Associated with Atherosclerosis in Patients with Transient Ischemic Attack or Ischemic Stroke. J Diabetes Metab 6: 496. doi:10.4172/2155-6156.1000496

Page 2 of 6

newly-diagnosed disturbed glucose metabolism, determined with fasting plasma glucose, 2-hour post-load glucose and glycosylated hemoglobin levels is associated with craniocervical atherosclerosis in patients with TIA or ischemic stroke compared with patients with preexistent diabetes mellitus or normal glucose metabolism.

\section{Research Design and Methods}

\section{Study population}

Patients were derived from the Erasmus Stroke Study, a registry of patients with cerebrovascular diseases treated at the Erasmus Medical Center Rotterdam. All consecutive patients with a clinical diagnosis of acute ischemic stroke or TIA between December 2005 and October 2010 were included. Patients without a CT-angiography and/or missing glucose assessment were excluded. Our study was approved by the Institutional Ethics Committee and written informed consent was obtained from all participants.

Demographic data, vascular history (previous TIA or ischemic stroke and previous ischemic heart disease among others) and risk factors (current smoking and BMI among others), laboratory assessments, and data on event characteristics were collected. Hypertension was defined as the use of antihypertensive drugs prior to the event.

\section{Glucose assessment}

Pre-existent diabetes mellitus was defined as the use of oral and/ or parenteral antidiabetic drugs prior to the TIA or ischemic stroke. In all patients, fasting plasma glucose and glycosylated hemoglobin levels were assessed on the $2^{\text {nd }}$ or $3^{\text {rd }}$ day of admission or when the patient visited the outpatient clinic in case of TIA patients who were not hospitalized. Subsequently, an oral glucose tolerance test (OGTT) was performed according to the World Health Organization protocol in all patients without pre-existing diabetes mellitus [25]. After overnight fasting, patients drank a solution of 75 grams glucose in $150 \mathrm{~mL}$ water, and 2-hour post-load glucose levels were assessed. Newly-diagnosed disturbed glucose metabolism was defined as 2 or more disturbed glucose tests according to the American Diabetes Association: fasting plasma glucose level $\geq 5.6 \mathrm{mmol} / \mathrm{L}$, and/or 2-hour post-load glucose level $\geq 7.8 \mathrm{mmol} / \mathrm{L}$, and/or glycosylated hemoglobin level $\geq 39 \mathrm{mmol} /$ mol [3].

\section{$\mathrm{CT}$ acquisition and analysis}

CT Angiography (CTA) of the carotid artery was performed with a 16 slice, 64 slice or 128 slice multi-detector CT system (Sensation 16, Sensation 64, Definition, Definition AS+ or Definition Flash, Siemens Medical Solutions, Erlangen, Germany) using a standardized optimized contrast-enhanced CTA protocol $(120 \mathrm{kVp}, 180-200 \mathrm{mAs}$, collimation $16 \times 0.75 \mathrm{~mm} ; 32 \times 2 \times 0.6 \mathrm{~mm} ; 64 \times 2 \times 0.6 \mathrm{~mm}$, pitch $<1)$. The scan range extended from the ascending aorta to the intracranial circulation $(3 \mathrm{~cm}$ above the sella turcica). All patients received $80 \mathrm{ml}$ of contrast agent ( $320 \mathrm{mg} / \mathrm{mL}$ iodixanol, Visipaque, Amersham Health, Little Chalfont, $\mathrm{UK})$, followed by $45 \mathrm{ml}$ saline bolus chaser, both at an injection rate of 4 or $5 \mathrm{ml} / \mathrm{s}$. Image reconstructions were made with field of view of 120 $\mathrm{mm}$, matrix size $512 \times 512$, slice thickness 0.75 or $1.0 \mathrm{~mm}$, increment 0.4 $-1.0 \mathrm{~mm}$ and with an intermediate reconstruction algorithm.

Stenosis: The severity of stenosis in carotid bifurcations (within 3 $\mathrm{cm}$ proximal and distal of the bifurcation) was assessed with the North American Symptomatic Carotid Endarterectomy Trial (NASCET) criteria [26]. A stenosis of $50 \%$ or more was considered significant. An intraclass correlation coefficient (ICC) for the degree of stenosis was based on the ratings of 3 independent observers (S.F., A.v.D. \& E.v.d.H.) on 50 CT examinations: 0.96-0.99.

Volume of calcifications: Dedicated commercially available software (Syngo CalciumScoring, Siemens) was used to semiautomatically quantify calcifications in the aortic arch and both extracranial carotid arteries, expressed as calcification volume in $\mathrm{mm}^{3}$. The aortic arch was defined as the origin of the aortic arch to the first $1 \mathrm{~cm}$ of the common carotid arteries, the vertebral arteries and the subclavian arteries beyond the origin of the vertebral arteries. The carotid arteries were scored within $3 \mathrm{~cm}$ proximal and distal of the bifurcation. A threshold of 600 Hounsfield units (HU) was used to differentiate calcifications from contrast material in the lumen. A detailed description of the measurement is provided elsewhere [16].

A custom-made plug-in for the freely available software ImageJ (Rasband, National Institute of Mental Health, Bethesda, MD, available at http://rsb.info.nih.gov/ij) was used for quantification of intracranial internal carotid artery calcifications. The intracranial internal carotid artery comprised the horizontal segment of the petrous internal carotid artery to the top of the internal carotid artery. Due to the close relationship of calcium in the arterial wall to the skull base, the previous mentioned semi-automatic tool could not be used. With the plug-in, it is possible to draw regions of interest in axial multidetector (MD) CTA images and to calculate automatically the total number of pixels above a predefined threshold. The volume of the intracranial calcifications (in $\mathrm{mm}^{3}$ ) was calculated as the product of the number of pixels above the threshold (600 HU), the pixel size, and the increment [27]. ICC for extracranial and intracranial calcifications was assessed, based on the ratings of respectively 3 independent observers (S.F., A.v.D. \& T.Z.) on 35 CT examinations (ICC 1.00) and the ratings of 2 independent observers (S.F. \& T.Z.) on 40 CT examinations (ICC 0.99).

\section{Statistical analysis}

The analysis was carried out with STATA 12.1 statistical package (Statacorp, College Station, Texas). Missing variables were imputed with single imputation using relevant baseline patient characteristics and the outcome variable. In the analysis with severity of stenosis, we used the highest degree of stenosis in both carotid artery bifurcations. In the analysis with calcification as continuous measure, we used natural log-transformed values and added $1.0 \mathrm{~mm}^{3}$ to the nontransformed values to deal with participants with a calcium score of zero. Calcification volumes of the left and right side were summed for the extra- and intracranial carotid arteries separately.

We performed logistic and linear analyses to study the relation between glucose metabolism on the one hand and atherosclerosis on the other. Adjustments were made with a multivariable logistic and linear regression model that included age, sex, hypertension, dyslipidemia, previous TIA or ischemic stroke, previous ischemic heart disease, current smoking, and BMI.

Based on the previous conflicting results between men and women, we repeated these analyses in men and women separately. Furthermore, we tested the difference in the effect of glucose on atherosclerosis between men and women and between patients with and without cholesterol-lowering drugs use prior to the event with a test for interaction.

\section{Results}

Between December 2005 and October 2010, 1611 consecutive patients with a TIA or ischemic stroke were included in the Erasmus 
Citation: Fonville S, van Dijk AC, Zadi T, van den Herik EG, Lingsma HF, et al. (2015) Newly-Diagnosed Disturbed Glucose Metabolism is Associated with Atherosclerosis in Patients with Transient Ischemic Attack or Ischemic Stroke. J Diabetes Metab 6: 496. doi:10.4172/2155-6156.1000496

Stroke Study. Of these patients, 205 (13\%) were excluded because no glucose assessment was performed and 189 patients (12\%) were excluded because no CTA was done because of contrast allergy, renal failure, or because a duplex ultrasound or CTA was performed in another hospital. In the remaining 1217 patients, the mean age was 62 years (SD 14), and 642 (53\%) were men.

Of the 1217 patients, 623 patients (51\%) had normal glucose metabolism, 384 (32\%) had newly-diagnosed disturbed glucose metabolism, and 210 (17\%) had pre-existent diabetes mellitus. Patients with newly-diagnosed disturbed glucose metabolism or preexistent diabetes mellitus were older, more often had hypertension, dyslipidemia, previous TIA or ischemic stroke, previous ischemic heart disease, were less often current smokers, and had a higher BMI. These patients were more often diagnosed with ischemic stroke, and the ischemic strokes were more severe. Large artery disease according to the TOAST criteria was more often present in patient with newlydiagnosed disturbed glucose metabolism or pre-existent diabetes mellitus. The demographic and clinical characteristics of the patients are shown in Table 1 .

\section{Atherosclerosis}

Patients with newly-diagnosed disturbed glucose metabolism or pre-existent diabetes mellitus more often had a carotid artery stenosis $\geq 50 \%$, and higher calcification volumes in aortic arch, carotid bifurcations, and intracranial carotid arteries, compared to patients with normal glucose metabolism (Figure 1 and Table 2).

The test for interaction showed no significant difference in the effect of glucose metabolism on atherosclerosis between men and women
( $p$-values $>0.05$, data not shown), hence further analyses between men and women were not performed.

However, the test for interaction showed a significant difference in the effect of glucose metabolism on calcification volumes (in the aortic arch, carotid bifurcations and intracranial carotid arteries) between patients with and without the use of cholesterol-lowering drugs prior to the event ( $p$-values $<0.001)$, but not on stenosis $\geq 50 \%$ ( $p$-value 0.221 ). After adjusting for confounders, pre-existent diabetes mellitus was associated with stenosis $\geq 50 \%$, and calcification volume in the aortic arch in all patients; and with calcification volume in the carotid bifurcations and intracranial carotid arteries in patients without the use of cholesterol-lowering drugs. Also, newly-diagnosed disturbed glucose metabolism was associated with stenosis $\geq 50 \%$ (Table 3 ).

\section{Conclusion}

Our study shows that newly-diagnosed disturbed glucose metabolism in patients with a recent TIA or ischemic stroke is independently associated with more severe extracranial atherosclerosis, and this association equaled that of pre-existent diabetes mellitus. Also, the association between pre-existent diabetes mellitus and calcification volume was more pronounced in patients without cholesterol-lowering drugs use prior to the event.

In line with previous studies, we found that pre-existent diabetes mellitus is associated with atherosclerosis in patients with TIA or ischemic stroke [13-16,28,29]. Furthermore, we found that newlydiagnosed disturbed glucose metabolism after TIA or ischemic stroke is associated with atherosclerosis. Most previous studies that assessed the association between glucose levels and atherosclerosis were performed

\begin{tabular}{|c|c|c|c|c|}
\hline & $\begin{array}{l}\text { Normal glucose metabolism } \\
\qquad \mathrm{N}=623\end{array}$ & $\begin{array}{c}\text { Newly-diagnosed disturbed } \\
\text { glucose metabolism } \\
N=384\end{array}$ & $\begin{array}{l}\text { Pre-existent diabetes } \\
\text { mellitus } \mathrm{N}=210\end{array}$ & p-value \\
\hline \multicolumn{5}{|l|}{ Demographic data } \\
\hline Age, mean (SD) in years & $59(14)$ & $66(13)$ & $65(12)$ & $<0.001$ \\
\hline Men, n (\%) & $316(51)$ & $202(53)$ & $124(59)$ & 0.112 \\
\hline Caucasian, n (\%) & $492(82)$ & $296(82)$ & $123(63)$ & $<0.001$ \\
\hline \multicolumn{5}{|l|}{ Risk factors } \\
\hline Hypertension*, n (\%) & $263(42)$ & $233(61)$ & $153(73)$ & $<0.001$ \\
\hline $\begin{array}{l}\text { Use of cholesterol-lowering drugs prior to the event, } n \\
(\%)\end{array}$ & $153(25)$ & $131(34)$ & $126(60)$ & $<0.001$ \\
\hline Atrial fibrillation, $\mathrm{n}(\%)$ & $29(5)$ & $30(8)$ & $17(8)$ & 0.063 \\
\hline Current smoking, $\mathrm{n}(\%)$ & $228(37)$ & $99(26)$ & $48(23)$ & $<0.001$ \\
\hline $\mathrm{BMI}$, mean (SD) in $\mathrm{kg} / \mathrm{m}^{2 \dagger}$ & $26(3)$ & $27(4)$ & $28(4)$ & $<0.001$ \\
\hline \multicolumn{5}{|l|}{ Medical history } \\
\hline TIA/ischemic stroke, n (\%) & $139(22)$ & $102(27)$ & $77(37)$ & $<0.001$ \\
\hline Ischemic heart disease, $\mathrm{n}(\%)$ & $82(13)$ & $69(18)$ & $65(31)$ & $<0.001$ \\
\hline \multicolumn{5}{|l|}{ Event characteristics } \\
\hline TIA, n (\%) & $287(46)$ & $133(35)$ & $69(33)$ & $<0.001$ \\
\hline NIHSS, median (IQR) ${ }^{\ddagger}$ & $2(1-5)$ & $3(1-6)$ & $3(2-5)$ & 0.006 \\
\hline \multicolumn{4}{|l|}{ TOAST classification } & $<0.001$ \\
\hline Large artery disease, $\mathrm{n}(\%)$ & $82(13)$ & $94(25)$ & $39(19)$ & \\
\hline Cardio-embolism, n (\%) & $68(11)$ & $51(13)$ & $30(14)$ & \\
\hline Small vessel disease, $\mathrm{n}(\%)$ & $131(21)$ & $62(16)$ & $50(24)$ & \\
\hline Other determined disease, $\mathrm{n}(\%)$ & $47(8)$ & $12(3)$ & $7(3)$ & \\
\hline Undetermined disease, $\mathrm{n}(\%)$ & $295(47)$ & $165(43)$ & $84(40)$ & \\
\hline \multicolumn{5}{|l|}{ Glucose assessment } \\
\hline Fasting plasma glucose, mean (SD) in mmol/L & $4.9(0.6)$ & $6.2(1.4)$ & $7.9(3.0)$ & $<0.001$ \\
\hline 2-hour post-load glucose, mean (SD) in mmol/L & $7.5(2.4)$ & $11.2(3.6)$ & NA & $<0.001$ \\
\hline Glycosylated hemoglobin, mean (SD) in $\mathrm{mmol} / \mathrm{mol}$ & $36(4)$ & $43(8)$ & $58(17)$ & $<0.001$ \\
\hline
\end{tabular}

Table 1: Patient characteristics per glucose metabolism group ( ${ }^{*}$ defined as the use of antihypertensive drugs prior to the ischemic stroke/TIA, ${ }^{\dagger}$ missing in 490 patients, imputated BMI used, tin patients with ischemic stroke only). 
Citation: Fonville S, van Dijk AC, Zadi T, van den Herik EG, Lingsma HF, et al. (2015) Newly-Diagnosed Disturbed Glucose Metabolism is Associated with Atherosclerosis in Patients with Transient Ischemic Attack or Ischemic Stroke. J Diabetes Metab 6: 496. doi:10.4172/2155-6156.1000496

Page 4 of 6
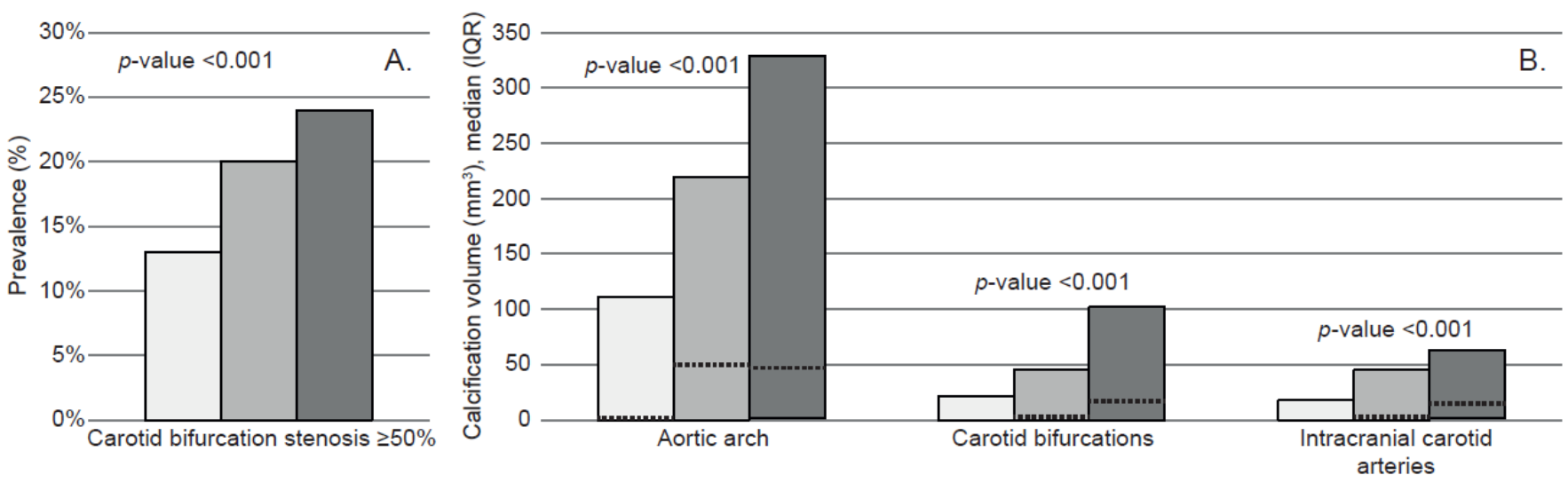

Figure 1: Atherosclerotic measurements in patients with normal glucose metabolism (light grey), newly-diagnosed disturbed glucose metabolism (medium grey) and pre-existent diabetes mellitus (dark grey): (A) prevalence of stenosis $\geq 50 \%$ in carotid bifurcation, (B) calcification volume (mm ${ }^{3}$, median (dashed line) and IQR) in aortic arch, carotid bifurcations, and intracranial carotid arteries.

\begin{tabular}{|c|c|c|c|c|}
\hline & $\begin{array}{l}\text { Normal glucose metabolism } \\
\qquad(n=623)\end{array}$ & $\begin{array}{l}\text { Newly-diagnosed disturbed } \\
\text { glucose metabolism } \\
(n=384)\end{array}$ & $\begin{array}{l}\text { Pre-existent diabetes mellitus } \\
\qquad(n=210)\end{array}$ & p-value \\
\hline Stenosis $\geq 50 \%, \mathrm{n}(\%)$ & $78(13)$ & $77(20)$ & $50(24)$ & $<0.001$ \\
\hline $\begin{array}{l}\text { Calcification volume in aortic arch, median (IQR) } \\
\text { in } \mathrm{mm}^{3}\end{array}$ & $3(0-111)$ & $50(0-219)$ & $48(1-329)$ & $<0.001$ \\
\hline $\begin{array}{l}\text { Calcification volume in carotid bifurcations, } \\
\text { median (IQR) in } \mathrm{mm}^{3}\end{array}$ & $0(0-22)$ & $5(0-46)$ & $17(0-102)$ & $<0.001$ \\
\hline $\begin{array}{l}\text { Calcification volume intracranial carotid arteries, } \\
\text { median (IQR) in } \mathrm{mm}^{3}\end{array}$ & $0(0-18)$ & $5(0-45)$ & $16(1-63)$ & $<0.001$ \\
\hline
\end{tabular}

Table 2: Atherosclerosis measurements in different glucose groups.

\begin{tabular}{|c|c|c|c|c|}
\hline & $\begin{array}{c}\text { Stenosis } \geq 50 \% \\
\text { aOR* }^{*}(95 \% \mathrm{Cl})\end{array}$ & $\begin{array}{l}\text { Calcification volume aortic } \\
\text { arch } \\
\text { Adjusted beta coeff }{ }^{\dagger}(95 \% \mathrm{Cl})\end{array}$ & $\begin{array}{l}\text { Calcification volume carotid } \\
\text { bifurcations } \\
\text { Adjusted beta coeff }{ }^{\dagger}(95 \% \mathrm{Cl})\end{array}$ & $\begin{array}{l}\text { Calcification volume intracranial } \\
\text { carotid arteries } \\
\text { Adjusted beta coeff }(95 \% \mathrm{Cl})\end{array}$ \\
\hline \multicolumn{5}{|c|}{ Newly-diagnosed disturbed glucose metabolism vs. normal glucose metabolism } \\
\hline All patients & $1.51(1.04-2.20)$ & $0.13(-0.12-0.39)$ & $0.08(-0.15-0.31)$ & $0.12(-0.14-0.38)$ \\
\hline $\begin{array}{l}\text { Patients without cholesterol-lowering } \\
\text { drugs }\end{array}$ & NS & $0.25(-0.05-0.54)$ & $0.10(-0.16-0.35)$ & $0.10(-0.19-0.39)$ \\
\hline $\begin{array}{c}\text { Patients with cholesterol-lowering } \\
\text { drugs }\end{array}$ & NS & $-0.04(-0.52-0.44)$ & $0.06(-0.39-0.51)$ & $0.14(-0.38-0.66)$ \\
\hline \multicolumn{5}{|c|}{ Pre-existent diabetes mellitus vs. normal glucose metabolism } \\
\hline All patients & $1.65(1.06-2.58)$ & $0.49(0.18-0.81)$ & $0.50(0.22-0.79)$ & $0.57(0.25-0.88)$ \\
\hline $\begin{array}{l}\text { Patients without cholesterol-lowering } \\
\text { drugs }\end{array}$ & NS & $0.51(0.07-0.95)$ & $0.79(0.40-1.18)$ & $0.94(0.52-1.36)$ \\
\hline $\begin{array}{c}\text { Patients with cholesterol-lowering } \\
\text { drugs }\end{array}$ & NS & $0.54(0.05-1.03)$ & $0.30(-0.17-0.76)$ & $0.34(-0.18-0.87)$ \\
\hline
\end{tabular}

Table 3: Association between both newly-diagnosed disturbed glucose metabolism and pre-existent diabetes mellitus and atherosclerosis, assessed with multivariable logistic and linear regression analysis (*odds ratio with corresponding 95\% confidence interval adjusted for age, sex, hypertension, cholesterol-lowering drugs, current smoking, previous ischemic heart disease, previous TIA/ischemic stroke, BMI; tbeta coefficient with corresponding 95\% confidence interval adjusted for age, sex, hypertension, cholesterol-lowering drugs, current smoking, previous ischemic heart disease, previous TIA/ischemic stroke, BMI; NS no significant interaction between cholesterol-lowering drugs use and stenosis).

in population-based studies and measured CIMT with duplex ultrasound instead of stenosis degree and calcification volume with CTA. We used degree of stenosis and calcification volumes assessed with CTA as a proxy for atherosclerosis. Carotid artery stenosis is a well-known and important risk factor for ischemic stroke and a stenosis of $50 \%$ or more is therefore a good marker for atherosclerosis [30]. Furthermore, in large population-based studies calcification volume in aortic arch and extracranial carotid arteries was not only associated with vascular risk factors, but also with the presence of (silent) cerebral infarcts on MRI and prevalent stroke [16,27,31]. Also, diabetes mellitus and glucose levels are significantly associated with calcification growth in patients with a TIA or ischemic stroke [32].
In contrast with previous studies [16-20], we found no significant interaction between glucose and sex. We therefore conclude that there is no consensus on the role of sex in the association between disturbed glucose metabolism and atherosclerosis. It is known that women with diabetes mellitus have a three- to seven-fold increased risk for coronary heart disease, compared to a two- to three-fold increased risk in men with diabetes mellitus [33]. Therefore, it would be interesting to investigate the possible different effects of glucose metabolism on atherosclerosis in men and women.

Atherosclerosis is an important vascular complication in patients with diabetes mellitus. The pathogenesis of atherosclerosis in diabetes mellitus is multifactorial. Not only the co-existence of other 
Citation: Fonville S, van Dijk AC, Zadi T, van den Herik EG, Lingsma HF, et al. (2015) Newly-Diagnosed Disturbed Glucose Metabolism is Associated with Atherosclerosis in Patients with Transient Ischemic Attack or Ischemic Stroke. J Diabetes Metab 6: 496. doi:10.4172/2155-6156.1000496

Page 5 of 6

cardiovascular risk factors in patients with diabetes mellitus like hypertension and dyslipidemia, but also insulin resistance, endothelial dysfunction, dyslipidemia, chronic inflammation, procoagulability, and impaired fibrinolysis promote atherosclerosis $[21,22]$. In patients with pre-diabetes, these processes may already play a role. This is also reflected in the Oxford Plaque Study, in which histological features of symptomatic atherosclerotic carotid plaques were assessed in patients with impaired glucose tolerance and diabetes mellitus. Not only in patients with diabetes mellitus, but also in patients with impaired glucose tolerance, surface thrombus and plaque macrophages seemed to persist longer in plaques, compared to plaques from those with normal glucose tolerance [34]. A recently developed mouse model could be used to investigate the causal role of impaired glucose tolerance in the pathogenesis of atherosclerosis [35]. However, whether pre-diabetes is associated with atherosclerosis remains controversial [13-15,23,24,3638]. Two recent meta-analyses found an association between impaired glucose tolerance and a small increase in CIMT $[24,39]$.

Interestingly, we found that the association between pre-existent diabetes and calcification volume was true for patients without cholesterol-lowering drugs use prior to the event. This underlines the importance of cholesterol on the atherogenesis in these patients [40]. In patients with newly-diagnosed disturbed glucose metabolism this association was not found, which might indicate that cholesterol starts to play a role in patients with more overt diabetes. However, we should take into account that we did not use a pre-specified test for interaction, so our results should be interpreted as hypothesis-generating.

To our knowledge, this is the first study that assessed the association between newly-diagnosed disturbed glucose metabolism and atherosclerosis in patients with ischemic stroke or TIA. Strengths of this study are the large sample size, detailed clinical information, the use of three different glucose tests and two different methods to assess atherosclerosis.

Our study has also some limitations. The glucose tests were not repeated to rule out laboratory error and the acute phase effect. Nonetheless, according to the American Diabetes Association two different simultaneously disturbed glucose tests are sufficient to diagnose pre-diabetes and newly-diagnosed diabetes mellitus and repeating the tests is therefore not obligatory [3]. Due to the small sample size patients with newly-diagnosed diabetes and pre-diabetes were combined into 1 group, although it would be interesting to establish the difference in the severity of atherosclerosis between these groups. Third, we excluded patients with missing glucose assessment and missing CTA, which might compromise the generalizability of our findings. Reasons for missing glucose assessments were mainly the poor medical condition of the patient, and these patients might therefore benefit less of secondary stroke prevention.

In conclusion, newly-diagnosed disturbed glucose metabolism, just like pre-existent diabetes mellitus, is independently associated with atherosclerosis in patients after ischemic stroke or TIA. This association may partly explain the increased risk for recurrent stroke in these patients. This study therefore might give a rationale to treat patients with newly-diagnosed disturbed glucose metabolism after ischemic stroke or TIA more aggressively to lower glucose levels and with cholesterol-lowering drugs. Future studies may include the effects of treatment on the progression of atherosclerosis in these patients.

\section{Acknowledgements}

S.F. researched data and wrote the manuscript. A.v.D. researched data. T.Z. researched data. Ev.d.H. researched data. H.L. reviewed the manuscript and contributed to the discussion. P.K. reviewed the manuscript and contributed to the discussion. A.v.d.L. reviewed the manuscript and contributed to the discussion. H.d.H. wrote the manuscript

\section{References}

1. Goldstein LB, Bushnell CD, Adams RJ, Appel LJ, Braun LT, et al. (2011) Guidelines for the primary prevention of stroke: a guideline for healthcare professionals from the American Heart Association/American Stroke Association. Stroke 42: 517-584.

2. Buysschaert M, Bergman M (2011) Definition of prediabetes. Med Clin North Am 95: 289-297, vii.

3. American Diabetes Association (2013) Diagnosis and classification of diabetes mellitus. Diabetes Care 36 Suppl 1: S67-74

4. Shaw J (2011) Diagnosis of prediabetes. Med Clin North Am 95: 341-352, viii.

5. Jia Q, Zheng H, Zhao X, Wang C, Liu G, et al. (2012) Abnormal glucose regulation in patients with acute stroke across China: prevalence and baseline patient characteristics. Stroke 43: 650-657.

6. Kernan WN, Viscoli CM, Inzucchi SE, Brass LM, Bravata DM, et al. (2005) Prevalence of abnormal glucose tolerance following a transient ischemic attack or ischemic stroke. Arch Intern Med 165: 227-233.

7. Matz K, Keresztes K, Tatschl C, Nowotny M, Dachenhausen A, et al. (2006) Disorders of glucose metabolism in acute stroke patients: an underrecognized problem. Diabetes Care 29: 792-797.

8. Gray CS, Scott JF, French JM, Alberti KG, O'Connell JE (2004) Prevalence and prediction of unrecognised diabetes mellitus and impaired glucose tolerance following acute stroke. Age Ageing 33: 71-77.

9. Fonville S, Zandbergen AA, Vermeer SE, Dippel DW, Koudstaal PJ, et al. (2013) Prevalence of prediabetes and newly diagnosed diabetes in patients with a transient ischemic attack or stroke. Cerebrovasc Dis 36: 283-289.

10. Ford ES, Zhao G, Li C (2010) Pre-diabetes and the risk for cardiovascular disease: a systematic review of the evidence. J Am Coll Cardiol 55: 1310-1317.

11. Lee M, Saver JL, Hong KS, Song S, Chang KH, et al. (2012) Effect of prediabetes on future risk of stroke: meta-analysis. BMJ 344: e3564.

12. Vermeer SE, Sandee W, Algra A, Koudstaal PJ, Kappelle LJ, et al. (2006) Impaired glucose tolerance increases stroke risk in nondiabetic patients with transient ischemic attack or minor ischemic stroke. Stroke 37: 1413-1417.

13. De Michele M, Panico S, Celentano E, Covetti G, Intrieri M, et al. (2002) Association of impaired glucose homeostasis with preclinical carotid atherosclerosis in women: Impact of the new American Diabetes Association criteria. Metabolism 51: 52-56.

14. Tropeano Al, Boutouyrie P, Katsahian S, Laloux B, Laurent S (2004) Glucose level is a major determinant of carotid intima-media thickness in patients with hypertension and hyperglycemia. J Hypertens 22: 2153-2160.

15. Brohall G, Odén A, Fagerberg B (2006) Carotid artery intima-media thickness in patients with Type 2 diabetes mellitus and impaired glucose tolerance: a systematic review. Diabet Med 23: 609-616.

16. Odink AE, van der Lugt A, Hofman A, Hunink MG, Breteler MM, et al. (2010) Risk factors for coronary, aortic arch and carotid calcification; The Rotterdam Study. J Hum Hypertens 24: 86-92.

17. Bos D, van der Rijk MJ, Geeraedts TE, Hofman A, Krestin GP, et al. (2012) Intracranial carotid artery atherosclerosis: prevalence and risk factors in the general population. Stroke 43: 1878-1884

18. Iribarren C, Sidney S, Sternfeld B, Browner WS (2000) Calcification of the aortic arch: risk factors and association with coronary heart disease, stroke and peripheral vascular disease. JAMA 283: 2810-2815.

19. Reilly MP, Wolfe ML, Localio AR, Rader DJ (2004) Coronary artery calcification and cardiovascular risk factors: impact of the analytic approach. Atherosclerosis 173: 69-78.

20. Hoff JA, Daviglus ML, Chomka EV, Krainik AJ, Sevrukov A, et al. (2003) Conventional coronary artery disease risk factors and coronary artery calcium detected by electron beam tomography in 30,908 healthy individuals. Ann Epidemiol 13: 163-169.

21. Eringa EC, Serne EH, Meijer RI, Schalkwijk CG, Houben AJ, et al. (2013) 
Citation: Fonville S, van Dijk AC, Zadi T, van den Herik EG, Lingsma HF, et al. (2015) Newly-Diagnosed Disturbed Glucose Metabolism is Associated with Atherosclerosis in Patients with Transient Ischemic Attack or Ischemic Stroke. J Diabetes Metab 6: 496. doi:10.4172/2155-6156.1000496

Endothelial dysfunction in (pre)diabetes: characteristics, causative mechanisms and pathogenic role in type 2 diabetes. Rev Endocr Metab Disord 14: 39-48.

22. Rask-Madsen C, King GL (2013) Vascular complications of diabetes: mechanisms of injury and protective factors. Cell Metab 17: 20-33.

23. Zhang YF, Hong J, Zhan WW, Li XY, Gu WQ, et al. (2006) Hyperglycaemia after glucose loading is a major predictor of preclinical atherosclerosis in nondiabetic subjects. Clin Endocrinol (Oxf) 64: 153-157.

24. Brohall G, Schmidt C, Behre CJ, Hulthe J, Wikstrand J, et al. (2009) Association between impaired glucose tolerance and carotid atherosclerosis: a study in 64-year-old women and a meta-analysis. Nutr Metab Cardiovasc Dis 19: 327 333.

25. WHO, Definition (1999) Diagnosis and Classification of Diabetes Mellitus and Its Complications: Report of a WHO Consultation. Geneva, World Health Or.

26. North American Symptomatic Carotid Endarterectomy Trial Collaborators (1991) Beneficial effect of carotid endarterectomy in symptomatic patients with high-grade carotid stenosis. N Engl J Med 325: 445-453.

27. Bos D, Ikram MA, Elias-Smale SE, Krestin GP, Hofman A, et al. (2011) Calcification in major vessel beds relates to vascular brain disease. Arterioscler Thromb Vasc Biol 31: 2331-2337.

28. Homburg PJ, Plas GJ, Rozie S, van der Lugt A, Dippel DW (2011) Prevalence and calcification of intracranial arterial stenotic lesions as assessed with multidetector computed tomography angiography. Stroke 42: 1244-1250.

29. He C, Gu M, Jiang R, Li JH (2013) Noninvasive assessment of the carotid and cerebrovascular atherosclerotic plaques by multidetector $\mathrm{CT}$ in type-2 diabetes mellitus patients with transient ischemic attack or stroke. Diabetol Metab Syndr 5: 9.

30. Rothwell PM, Eliasziw M, Gutnikov SA, Fox AJ, Taylor DW, et al. (2003) Analysis of pooled data from the randomised controlled trials of endarterectomy for symptomatic carotid stenosis. Lancet 361: 107-116.

31. Elias-Smale SE, Odink AE, Wieberdink RG, Hofman A, Hunink MG, et al. (2010) Carotid, aortic arch and coronary calcification are related to history of stroke: the Rotterdam Study. Atherosclerosis 212: 656-660.
32. van Gils MJ, Bodde MC, Cremers LG, Dippel DW, van der Lugt A (2013) Determinants of calcification growth in atherosclerotic carotid arteries; a seria multi-detector CT angiography study. Atherosclerosis 227: 95-99.

33. Roeters van Lennep JE, Westerveld HT, Erkelens DW, van der Wall EE (2002) Risk factors for coronary heart disease: implications of gender. Cardiovasc Res 53: 538-549.

34. Redgrave JN, Lovett JK, Syed AB, Rothwell PM (2008) Histological features of symptomatic carotid plaques in patients with impaired glucose tolerance and diabetes (oxford plaque study). Cerebrovasc Dis 26: 79-86.

35. Asai A, Nagao M, Kawahara M, Shuto Y, Sugihara H, et al. (2013) Effect of impaired glucose tolerance on atherosclerotic lesion formation: An evaluation in selectively bred mice with different susceptibilities to glucose intolerance. Atherosclerosis 23: 421-426.

36. Bonora E, Kiechl S, Willeit J, Oberhollenzer F, Egger G et al. (1999) Plasma glucose within the normal range is not associated with carotid atherosclerosis prospective results in subjects with normal glucose tolerance from the Bruneck Study. Diabetes Care 22: 1339-1346.

37. Bonora E, Kiechl S, Oberhollenzer F, Egger G, Bonadonna RC, et al. (2000) Impaired glucose tolerance, Type II diabetes mellitus and carotid atherosclerosis: prospective results from the Bruneck Study. Diabetologia 43 156-164.

38. Emerging Risk Factors Collaboration, Sarwar N, Gao P, Seshasai SR, Gobin $R$, et al. (2010) Diabetes mellitus, fasting blood glucose concentration, and risk of vascular disease: a collaborative meta-analysis of 102 prospective studies Lancet 375: 2215-2222.

39. Einarson TR, Hunchuck J, Hemels M (2010) Relationship between blood glucose and carotid intima media thickness: A meta-analysis. Cardiovasc Diabetol 9: 37.

40. Turner RC, Millns H, Neil HA, Stratton IM, Manley SE, et al. (1998) Risk factors for coronary artery disease in non-insulin dependent diabetes mellitus: United Kingdom Prospective Diabetes Study (UKPDS: 23) BMJ 316: 823-828. 